26

\title{
Structured representations in a quantum probability model of similarity
}

\author{
Emmanuel M. Pothos ${ }^{1}$ \& Jennifer S. Trueblood ${ }^{2}$
}

Affiliations, correspondence: 1: Department of Psychology, City University London, London, EC1V OHB, UK, emmanuel.pothos.1@city.ac.uk; 2: Department of Cognitive Sciences, 3151 Social Sciences Plaza, University of California, Irvine, Irvine, CA 92697-5100, USA, jstruebl@uci.edu.

Word count: 7965; Running head: structure in similarity 


\section{Abstract}

Recently, Busemeyer et al. (2011) presented a model for how the conjunction fallacy (Tversky \& Kahneman, 1983) emerges, based on the principles of quantum probability (QP) theory. Pothos et al.

31 (2013) extended this model to account for the main similarity findings of Tversky (1977), which have

32 served as a golden standard for testing novel theories of similarity. However, Tversky's (1977) empirical

33 findings did not address the now established insight that, in comparing two objects, overlap in matching 34 parts of the objects tends to have a greater impact on their similarity, than overlap in non-matching 35 parts. We show how the QP similarity model can be directly extended to accommodate structure in 36 similarity comparisons. Smolensky's et al.'s (in press) proposal for modeling structure in linguistic 37 representations, with tensor products, can be adapted 'as is' with the QP similarity model. The formal 38 properties of the extended QP similarity model are analyzed, some indicative fits are presented, and, 39 finally, a novel prediction is developed.

41 keywords: quantum probability theory, similarity, representation 
1. Introduction

57

We call quantum probability (QP) theory the rules for assigning probability to events, without any of the physics (Hughes, 1989, Isham, 1989). QP theory is a framework for probabilistic inference alternative to that of classical probability (CP) theory. A case for adopting QP theory, instead of classical probability (CP) theory, in cognitive modeling has been made when human behavior appears at odds with the prescription from CP theory (e.g., Aerts, 2009; Aerts \& Gabora, 1995; Blutner et al., 2013; Nelson et al., in press; for overviews see Busemeyer \& Bruza, 2012; Haven \& Khrennikov, 2013; Khrennikov, 2010; Pothos \& Busemeyer, 2013; Wang et al., 2013; Yearsley \& Pothos, 2014). Recently, Busemeyer et al. (2011) presented a model of decision making, based on QP principles, with an emphasis on how the conjunction fallacy (Tversky \& Kahneman, 1983), and related findings, emerge. For example, in the conjunction fallacy experiment, participants were told of a hypothetical person, Linda, described very much as a feminist (F) and not at all as a bank teller (BT). Participant responses indicated that $\operatorname{Prob}(F \wedge B T)>\operatorname{Prob}(B T)$, which is impossible classically. In Busemeyer at el.'s (2011) QP model for this conjunction fallacy, if one assumes that the $B T, F$ possibilities are incompatible, then it can emerge that the quantum probability of $F \wedge$ then $B T$ is higher than that of $B$.

Pothos, Busemeyer, and Trueblood (2013) considered whether the QP decision model could be extended to account for basic similarity judgments. Their motivation was that QP theory is formalized in multidimensional, vector spaces, called Hilbert spaces. The most common, standard way to model basic similarity involves multidimensional representations (e.g., Shepard, 1987) and the conceptualization of similarity as a function of distance. For example, such models have been employed in the predominant approaches to categorization (e.g., Goldstone, 1994a; Nosofsky, 1984; Wills \& Pothos, 2012). Therefore, since QP representations are also geometric (that is, involve elements in some multidimensional vector space), perhaps QP theory can provide some interesting generalization to the standard distance-based similarity models?

Note first that, by basic similarity judgments, we imply ones that are nonanalytic (in the psychological sense), direct, and immediate. If we accept the view that basic similarity judgments can be modeled as some function of distance, then they have to be consistent with the metric axioms mathematical requirements that all (simple) functions of distances need to obey. These axioms are intuitively appealing. For example, the symmetry axiom, requires that $\operatorname{distance}(A, B)=\operatorname{distance}(B, A)$, implying that $\operatorname{similarity}(A, B)=\operatorname{similarity}(B, A)$. In one of the most influential studies in the basic similarity literature, Tversky (1977) showed that all metric axioms can be violated in similarity judgments of naïve observers. Tversky's (1977) findings have become a golden standard of empirical results that should be accounted for by any basic similarity model and, indeed, have been the focus of theoretical effort in related research ever since (e.g., Ashby \& Perrin, 1988; Krumhansl, 1978). It is worth noting that basic, distance-based similarity metrics can be made to violate the metric axioms. For example, symmetry can be violated if $\operatorname{similarity}(A, B)=p_{A B}$. distance $(A, B)$, where $p_{A B}$ is just a directionality parameter, that is a parameter which can have a different value depending on whether the similarity evaluated is between $A$ and $B$ or between $B$ and $A$ 
(Nosofsky, 1991). However, the real challenge has been to explore how consistency with Tversky's (1977) findings can emerge from the structure of a basic similarity model.

Pothos et al. (2013) showed how the QP decision model can indeed accommodate Tversky's (1977) key findings, with fairly minor modifications. The objects to be compared in a similarity judgment are represented as subspaces, whose dimensionality depends on the extent of knowledge we have about the objects. Then, similarity judgments are modeled just as conjunctive probabilities of thinking of the first compared object and then the second (one also needs to assume a relevant mental state, that is neutral between the compared objects), that is, $\operatorname{Sim}(A, B)=\operatorname{Prob}(A \wedge$ then $B)$ (see also Section 2$)$. For example, Tversky's (1977) famous example of violations of symmetry in similarity judgments was the finding that $\operatorname{Sim}($ Korea, China) $>\operatorname{Sim}($ China,Korea), given that participants have more extensive knowledge for China, than Korea (note, actually Red China and North Korea). In the QP model similarity model, this asymmetry can emerge, as long as the dimensionality for the China subspace is greater than that for the Korea subspace.

The application of the QP decision model onto similarity indicates that the formalism can encompass findings from both decision making and basic similarity. Clearly, such a statement needs to be qualified, since the decision QP model addresses only certain kinds of decision making results and, likewise, the similarity one, only certain kinds of basic similarity results. Nevertheless, Pothos et al.'s theory (2013) is encouraging and fits with an overall prerogative to explain as wide a range of empirical findings as possible, with as few explanatory principles as possible. For example, supporting the same QP model in both decision making and basic similarity makes it plausible that the same principles underlie both kinds of cognitive processes.

While our focus until now has been on basic similarly, a (somewhat) separate literature has evolved around analogical similarity, which partly concerns the study of analogy formation. Simplifying, analogy formation is about how a naïve observer can establish associations between the elements of two representations (e.g., the atom and the solar system). From a cognitive process point of view, analogy is sometimes analytic and involved (cf. Casale et al., 2012), in contrast to basic similarity. A key focus for models of analogical similarity has been the correspondence between the constituent elements of two compared objects; how do they develop and what is their role in the overall similarity judgment, between the compared objects (Gentner, 1983; Goldstone, 1994b; Goldstone \& Son, 2005; Larkey \& Markman, 2005; Taylor \& Hummel, 2009)? For example, suppose we are comparing two persons, Sue and Linda, with black hair. Surely, this fact would contribute more to the overall similarity judgment between Sue and Linda, compared to an alternative situation, where Sue has black hair and Linda has black shoes. That is, there is intuition and supporting evidence that human similarity judgments are sensitive to the structure of the compared objects. An influential idea in modeling structure in similarity judgments is that feature matches can be aligned or not aligned (Goldstone, 1994b; cf. Markman \& Gentner, 1993). That is, parts of one object can be placed in correspondence with the parts of another or not. The implication is that matching aligned parts have a greater impact on similarity judgments than matching unaligned parts, but the latter can increase similarity too.

The purpose of this work is to examine whether the QP basic similarity model can be further extended to cover some key requirements for analogical similarity, notably the way similarity computations are affected by correspondences between representation parts (we do not consider the mechanisms that lead to the discovery of which features align or not; this is an important aspect of 
research in analogical similarity, but beyond the scope of this work). An extension of this sort cannot be expected to perform as well on analogical similarity results, as thoroughbred models of analogical similarity (e.g., Goldstone, 1994b; Hahn et al., 2009; Larkey \& Markman, 2005). Nevertheless, attempting the extension is important: if successful, it will show that the mathematical principles guiding basic similarity judgments are (plausibly and to some extent) the same as the ones underlying analogical similarity judgments (though equally, if the general model leads to inferior fits in the novel domain, perhaps a restricted scope is more appropriate). This question of possible equivalence of guiding principles is separate from the one concerning brain systems (cf. Casale et al., 2012). Note also that most basic similarity models cannot account for structure in similarity judgments (Tversky, 1977, Ashby \& Perrin, 1988; Krumhansl, 1978; we return to this point later). Likewise, current attempts to extend analogical similarity models to cover Tversky's (1977) results have difficulties. The separatedness of the literatures on basic similarity and analogical similarity further motivate the present effort to develop a QP model of basic/analogical similarity.

As it turns out, there is a very straightforward way to extend the QP model of basic similarity into one of analogical similarity, using Smolensky et al.'s (in press; Smolensky, 1990) ideas for modeling structure in linguistic representations. They were interested in the similarity between linguistic representations, where the role in one representation was compared to the role in the other, and likewise for the fillers (e.g., in relation to phonology, a role could be 'syllable-onset' and a corresponding filler could be ' $r$ ', for the word 'rat', as in Smolensky at et.'s, in press, example, on p.5). They derived their similarity method by employing a tensor product representation, which effectively separated out information of different kinds (roles, fillers), in separate vector spaces. Smolensky et al.'s ideas can be applied to the QP similarity model more or less as they are (since tensor products are standard in QP representations), thus revealing an interesting convergence between QP modeling and computational tools in prior, totally unrelated work. Does the resulting QP model of basic/analogical similarity have any interesting or unique properties? We take up this challenge in relation to possible interference in the contributions to similarity, from different parts of the compared objects. We also provide some indicative fits of the QP model, showing that, with a reasonable parameterization, the performance of the model is acceptable.

\section{Quantum probability theory in cognitive modeling and the quantum similarity model}

We briefly outline the QP model of basic similarity (for a more complete presentation and the application of the model to Tversky's, 1977, findings see Pothos et al., 2013). The QP basic similarity model is based on a unit length state vector representing mental states, which exists in a Hilbert space. Subspaces of varying dimensionality represent the instances, objects, or concepts, which are to be compared. Each subspace is associated with a linear operator, called a projector. Projectors are fundamental in QP theory. Their function is to take a vector and 'lay it down' onto a corresponding subspace. Suppose we are interested in the similarity between objects $A$ and $B$, such that the projectors to the corresponding subspaces are $P_{A}$ and $P_{B}$. The mental state vector just prior to the comparison is 
denoted as $|\psi\rangle$, in Dirac bra-ket notation, whereby $|x\rangle$ denotes a column vector and $|x\rangle^{\prime}$ the complex conjugate of the transpose of $|x\rangle$ (and so a row vector). Then, according to the quantum similarity model, just prior to a comparison, the state vector is assumed to be neutral between the compared objects. For example, when comparing objects $A$ and $B$, we assume $\| P_{B} \cdot|\psi\rangle\left\|^{2}=\right\| P_{A} \cdot|\psi\rangle \|^{2}{ }^{2}{ }^{1}$ This condition $\left.\left.\left|P_{A} \cdot\right| \psi\right\rangle\left.\right|^{2}=\left|P_{B} \cdot\right| \psi\right\rangle\left.\right|^{2}$ on the state vector is equivalent to that of an uninformed Bayesian prior and basically means that the mental state prior to comparing objects $A$ and $B$ is such that it favors neither $A$ nor $B$. As noted, $\operatorname{Sim}(A, B)=\operatorname{Prob}(A \wedge$ then $B)$, which is given by $\operatorname{Sim}(A, B)=\mid P_{B} \cdot P_{A}$. $\left.|\psi\rangle\right|^{2}$; this is the squared length of the projection of the state vector, from the subspace of one compared entity, to the next. The specific interpretation of probability is that that the state vector is consistent with the first possibility and then the second (note that, a remarkable theorem by Gleason, 1957, shows that the QP probability rule, for how to go from projections to probabilities, is unique).

As a simple illustration of the quantum similarity model, consider the case where $A$ and $B$ both correspond to unidimensional subspaces in a two-dimensional space. Let $|A\rangle$ and $|B\rangle$ be normalized column vectors along the $A$ and $B$ subspaces respectively. Then, the complex conjugate transpose vectors would be denoted as $\langle A|$ and $\langle B|$. The corresponding projector operators are easily defined as, e.g., $|A\rangle\langle A|$. If $|A\rangle=\left[\begin{array}{l}a 1 \\ a 2\end{array}\right]$, then $P_{A}=|A\rangle\langle A|=\left[\begin{array}{l}a 1 \\ a 2\end{array}\right]\left[\begin{array}{ll}a 1^{*} & a 2^{*}\end{array}\right]$, which is a $2 \times 2$ matrix. Also, $\langle A \mid A\rangle=$ $\left[\begin{array}{ll}a 1^{*} & a 2^{*}\end{array}\right]\left[\begin{array}{l}a 1 \\ a 2\end{array}\right]=a 1^{*} \cdot a 1+a 2^{*} \cdot a 2$, that is, $\langle A \mid A\rangle$ is a dot product. With the above definition of similarity we have $\left.\left.\operatorname{Sim}(A, B)=\left|P_{B} \cdot P_{A} \cdot\right| \psi\right\rangle\left.\right|^{2}=|| B\right\rangle\left.\langle B|\cdot| A\rangle\langle A|\cdot| \psi\rangle\right|^{2}=|\langle A \mid B\rangle|^{2}|\langle A \mid \psi\rangle|^{2}$ and $\operatorname{Sim}(B, A)=|\langle A \mid B\rangle|^{2}|\langle B \mid \psi\rangle|^{2}$, noting that the norm of $|A\rangle$ and $|B\rangle$ is 1 and that $|\langle A \mid B\rangle|^{2}=|\langle B \mid A\rangle|^{2}$. With the assumption that $|\langle B \mid \psi\rangle|^{2}=|\langle A \mid \psi\rangle|^{2}$, the relative similarity between $A$ and $B$ is effectively based on $|\langle A \mid B\rangle|^{2}$. In a real space, $\langle A \mid B\rangle=|A| \cdot|B| \cdot \cos \theta$, where $\theta$ is the angle between the $|A\rangle$ and $|B\rangle$ vectors (normalization further means that $|A|=|B|=1$ ). Thus, overall, $\operatorname{Sim}(A, B) \propto(\cos \theta)^{2}$, that is, at least for objects represented with one-dimensional subspaces, similarity is just a decreasing function of the angle between the subspaces (up to $\theta=\frac{\pi}{2}$ ). In brief, this explains how the quantum similarity model works and the basic intuition that drives its formulation.

\section{Extending to the QP model of basic similarity to analogical similarity}

We introduce the analogical extension to the QP model of basic similarity, using a standard example of analogical similarity, from Goldstone (1994b). In one of his experiments, participants were asked to compare drawings of birds with four distinct parts, left wing, right wing, body, and head (Figure 1). Each part would include a specific feature (one of 21 random symbols). The features for the four parts can be denoted with four letters, such as ABCD. Thus, a comparison in Goldstone's (1994b) experiment can be denoted as $A B C D$ vs. BADC. In this particular comparison, the two birds share four features, but the shared features do not correspond to the same parts of the birds. Goldstone (1994b) called such matches MOPs, that is, matches out of place. By analogy, MIPs, matches in place, are shared features for

\footnotetext{
${ }^{1}$ Note, the double lines indicate the norm of the projected vector and single lines the modulus of a complex number. As in all cases, we are dealing with normalized vectors, norms such as the above resolve to computing the modulus of dot products. So, to simplify notation, henceforth we will just write e.g. $\left.\left|P_{A} \cdot\right| \psi\right\rangle\left.\right|^{2}$.
} 
the same parts of the bird. Thus, the two representations $A B C D$ and $A B X Z$ would have two MIPs. The distinction between MIPs and MOPs captures a fundamental aspect of structure in representations, namely the fact that particular features could be shared between two objects, without corresponding to the same part of the objects. Goldstone (1994b) found that MIPs have more of an impact on similarity than MOPs, but both MIPs and MOPs increase similarity. He also reported that having both a MIP and a MOP for the same feature (e.g., AACD compared to ABXZ) would actually lead to a lower rating, than just a corresponding MIP (e.g., AYXZ). Specifically, in his Experiment 2 (stimuli were schematic birds with symbols), the similarity result for comparing objects with three MIPs was higher than the result with three MIPs and one MOP (Figure 7, p.11). Goldstone (1994b) considered this result important enough to highlight the ability of his model, (Similarity as Interactive Activation and Mapping; SIAM) to capture it, against competitors. But, the corresponding difference was not significant and not replicated in Larkey and Markman (2005). Finally, Note that there is a related distinction in the literature, between aligned and unaligned differences (e.g., Markman \& Gentner, 1996). The consideration of similarity using aligned and unaligned differences is not quite equivalent to that of MIPs and MOPs but, as far as we can see, there are no relevant implications in the discussion below.
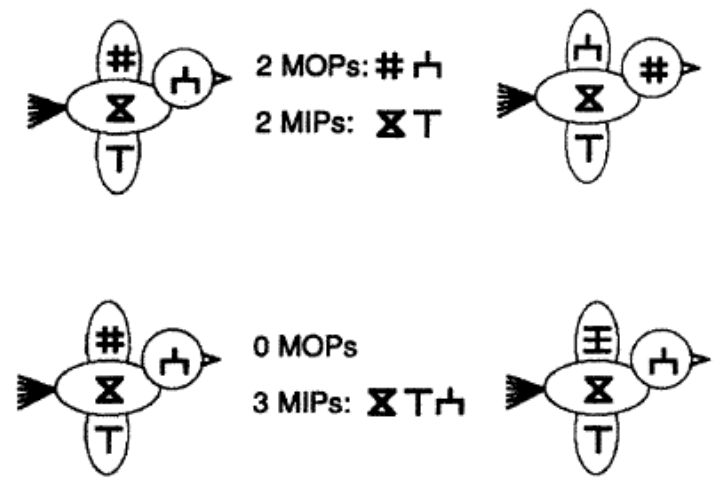

Figure 1. A figure reproduced from Goldstone (1994b; Figure 6), illustrating the stimuli employed in his Experiment 2. [Note to copy editor: according to APA guidelines, permission is not required for reproducing a maximum of three figures or tables from a journal article.]

Smolensky et al. (in press) solved the problem of introducing structure into geometric representations in the context of combinatorial structure in connectionist models of language processing. Specifically, his starting point (Smolensky et al., in press) was two structured representations $S$ and $S^{\prime}$, which were composed of roles (denoted by $r$ ) and fillers for each role (denoted by $f$ ). In other words, $S=\left\{f_{j} / r_{j}\right\}_{j}$ and $S^{\prime}=\left\{f^{\prime}{ }_{k} / r^{\prime}{ }_{k}\right\}_{k}$, whereby $\left\{f_{j} / r_{j}\right\}_{j}$ denotes a set of roles and corresponding fillers. For example, $S$ and $S$ ' might be two different syllables, $r$ different roles, such as 'onset' or 'coda', and $f$ possible fillers, i.e., phonemes. Then, Smolensky et al. suggested that the similarity between these structured representations should be given by a scheme which computes $\operatorname{sim}\left(S, S^{\prime}\right)=\sum_{j} \sum_{k} \operatorname{sim}\left(f_{j}, f^{\prime}{ }_{k}\right) \cdot \operatorname{sim}\left(r_{j}, r^{\prime}{ }_{k}\right)$, that is, a scheme which computes the similarity between the corresponding fillers, for all combinations of roles. Smolensky (1990; Smolensky et al., in press) noted that one way to implement this similarity measure is by considering the tensor product between roles and fillers and it is this approach which we adopt in the QP model of basic/analogical similarity. Note that other researchers have employed Smolensky's (1990) tensor product idea in analogy 
formation (e.g., Wiles et al., 1994; see also Humphreys et al., 1989), but placed less emphasis on the distinction between of MIPs and MOPs. In Wiles et al. (1994) work, tensors are employed to examine analogies between e.g. "mother: baby" and "mare: foal". Humphreys et al.'s (1989) model is a model of memory, whereby tensor products are employed to bind words with their contexts. Also, some researchers have pointed out that a tensor product scheme for combining roles and fillers may involve a combinatorial explosion of overall dimensionality. It's unclear how much a problem this is in practice. Smolensky et al. (in press) argued that it is not. Also, in similarity applications, a problem of overall dimensionality is likely to be less severe than in language.

What is the subset of possible role combinations allowed? This depends entirely on how the vectors for roles are set up. In the most general form for the similarity measure, $\operatorname{sim}\left(S, S^{\prime}\right)=$ $\sum_{j} \sum_{k} \operatorname{sim}\left(f_{j}, f^{\prime}{ }_{k}\right) \cdot \operatorname{sim}\left(r_{j}, r^{\prime}{ }_{k}\right)$, it should be clear that all combinations of roles are allowed, so that the similarity measure is consistent with both MIPs and MOPs.

We first illustrate our approach just for MIPs, for simple objects having three distinct parts, top, middle, and bottom (a part is like a role in Smolensky et al.'s example above). Each part can be any of two shapes, a square or a circle. Moreover, any shape can be any of two colors, say blue and red (the shapes and colors are possible fillers, for the roles above). Following Smolensky (1990; Smolensky et al., in press), we suggest that the set of all such objects would be represented by vectors of the form $\mid$ top part $\rangle \otimes \mid$ shape $\rangle \otimes \mid$ color $\rangle+\mid$ middle part $\rangle \otimes \mid$ shape $\rangle \otimes \mid$ color $\rangle+$ $\mid$ bottom part $\rangle \otimes \mid$ shape $\rangle \otimes \mid$ color $\rangle$. Note that in our approach the rank of the tensors depends on the number of elementary features, which together characterize an object part (for an alternative approach see Wiles et al., 1994). The role vectors indicate the part under consideration and, since we are assuming only MIPs, we can set these to a canonical basis to write

$$
\left.\left.\left.\left.\left.\left.\left(\begin{array}{l}
1 \\
0 \\
0
\end{array}\right) \otimes \mid \text { shape }\right\rangle \otimes \mid \text { color }\right\rangle+\left(\begin{array}{l}
0 \\
1 \\
0
\end{array}\right) \otimes \mid \text { shape }\right\rangle \otimes \mid \text { color }\right\rangle+\left(\begin{array}{l}
0 \\
0 \\
1
\end{array}\right) \otimes \mid \text { shape }\right\rangle \otimes \mid \text { color }\right\rangle
$$

The tensor product of the part vector, with the vector corresponding to shape, and the vector corresponding to color, allows all combinations of shapes and colors for each part.

In the QP basic similarity model, the similarity between two objects, $A$ and $B$, is $\operatorname{Sim}(A, B)=$ $\left.\left|P_{B} \cdot P_{A} \cdot\right| \psi\right\rangle\left.\right|^{2}$. In the extended QP model of basic/analogical similarity, the definition of similarity is identical. The similarity computation is straightforward to carry out. Note first that we can write $\operatorname{Sim}(A, B)=|| A\rangle\left[A|B\rangle\left[\left.B|\psi\rangle\right|^{2}\right.\right.$. The first dot product, $[B|\psi\rangle$, can be ignored, since we require the state vector, $|\psi\rangle$, to be neutral with respect to the $A$ and $B$ rays (i.e., $|\langle B \mid \psi\rangle|^{2}=|\langle A \mid \psi\rangle|^{2}$ ). The $|A\rangle$ term can be ignored as well, since ||$A\rangle\left.\right|^{2}=1$. Therefore, we can write $\operatorname{Sim}(A, B)=\left|\left[\left.A|B\rangle\right|^{2}\right.\right.$. The dot product is

$\Xi A|B\rangle=\left(\begin{array}{l}1 \\ 0 \\ 0\end{array}\right)_{A} \otimes(\text { shape })_{A} \otimes(\text { color })_{A}+\left(\begin{array}{l}0 \\ 1 \\ 0\end{array}\right)_{A} \otimes(\text { shape })_{A} \otimes(\text { color })_{A}+\left(\begin{array}{l}0 \\ 0 \\ 1\end{array}\right)_{A} \otimes(\text { shape })_{A} \otimes(\text { color })_{A} \mid$ $\left.\left(\begin{array}{l}1 \\ 0 \\ 0\end{array}\right)_{B} \otimes(\text { shape })_{B} \otimes(\text { color })_{B}+\left(\begin{array}{l}0 \\ 1 \\ 0\end{array}\right)_{B} \otimes(\text { shape })_{B} \otimes(\text { color })_{B}+\left(\begin{array}{l}0 \\ 0 \\ 1\end{array}\right)_{B} \otimes(\text { shape })_{B} \otimes(\text { color })_{B}\right\rangle$ 
This leads to

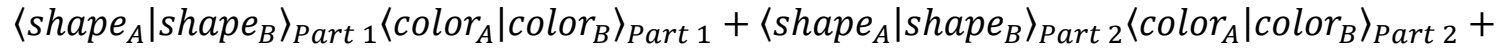
$\left\langle\right.$ shape $\left._{A}\right|$ shape $\left._{B}\right\rangle_{\text {Part }_{3}}\left\langle\right.$ color $\left._{A}\right|$ color $\left._{B}\right\rangle_{\text {Part } 3}$

The above computation is simpler than perhaps how it looks. All terms combine with each other and each individual dot product is of the form $\left[x \otimes y \otimes z\left|x^{\prime} \otimes y^{\prime} \otimes z^{\prime}\right\rangle=\left[x\left|x^{\prime}\right\rangle\right.\right.$ ? $\left|y^{\prime}\right\rangle\left[z\left|z^{\prime}\right\rangle\right.$. In other words, the dot product between two tensor products decomposes into dot products for each 'slot' (space) separately. The first of these dot products involves the vector for the particular parts under consideration in the two objects. But, these part vectors have been set up in such a way that, unless they are the same, their dot product is zero. For example, consider the dot product involving the first part of object $A$ and the second part of object $B$. This is

国 $\left.\left.\begin{array}{l}1 \\ 0 \\ 0\end{array}\right)_{A} \otimes(\text { shape })_{A} \otimes(\text { color })_{A} \mid\left(\begin{array}{l}0 \\ 1 \\ 0\end{array}\right)_{B} \otimes(\text { shape })_{B} \otimes(\text { color })_{B}\right\rangle=\left(\begin{array}{lll}1 & 0 & 0\end{array}\right)_{A}\left(\begin{array}{l}0 \\ 1 \\ 0\end{array}\right)_{B} \ldots=0$

So, overall, this particular way of setting up the part vectors readily allows for similarity comparisons to be faithful to structure, in the sense that shape equivalence adds to the similarity computation, only when the corresponding parts are the same between the two objects. In brief, this is a scheme for MIPs.

The QP model of basic/analogical similarity can easily allow for contributions to similarity from MOPs as well, in a way identical to that of Smolensky et al. (in press), by allowing non-orthogonal part vectors (i.e., non-orthogonal roles). Note that, in the QP model, MOPs can only arise from similarities between roles; if roles have no similarity structure, then the QP model must predict no MOPs. We are also now led to a key distinction between the QP model of basic/analogical similarity and Smolensky et al.'s (in press) approach. The native space of QP representations is complex. In QP theory, complex numbers are employed as a technical convenience and all outputs of the theory are guaranteed to be real. But, in part because of the use of complex vectors, QP theory computations sometimes give rise to interference effects, not possible with real vectors. Therefore, with non-orthogonal, complex vectors for roles, we are led to the possibility of not only MOPs, but of interference effects between MOPs and MIPs.

To illustrate, consider a simplified example of two objects created of two possible features in two possible roles, so that $A=r_{1} \otimes f_{1}+r_{2} \otimes f_{2}$ and $B=r_{1} \otimes f_{1}^{\prime}+r_{2} \otimes f_{2}^{\prime}$, noting that $r_{1}$ is the role vector for the first part and $r_{2}$ the role vector for the second part. The actual features $f_{1}, f_{2}$ etc. could be the same or not, this is not relevant presently. Then, $\operatorname{Sim}(A, B) \propto\left|\left[\left.A|B\rangle\right|^{2}\right.\right.$. The inner product is computed

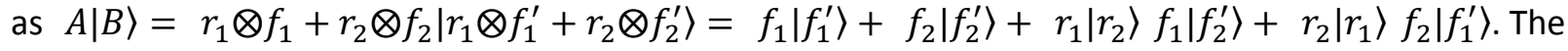
first two terms are contributions from MIPs; the cross-terms are MOPs. Here, we are only concerned with the cross-terms, since the MIPs contribution cannot change by altering the role vectors. We can rewrite the cross-terms as: cross $=q_{1}\left|r_{2}\right\rangle G+\sigma_{2}\left|r_{1}\right\rangle H$, where $G=\sigma_{1} f_{1}\left|f_{2}^{\prime}\right\rangle$ and $H=\sigma_{2}\left|f_{1}^{\prime}\right\rangle$. Depending on the form of $r_{1}, r_{2}$, there is potential for the cross-terms to reduce the overall value of $[A|B\rangle$, when the contributions to similarity from MOPs can be negative. This is simply illustrated in two dimensions. Letting $x, y, a, b$, be all positive, real numbers, consider four possibilities: 
$r_{1}=\left(\begin{array}{l}x \\ y\end{array}\right), r_{2}=\left(\begin{array}{l}a \\ b\end{array}\right)$, then $r_{1}\left|r_{2}\right\rangle G+\sigma_{2}\left|r_{1}\right\rangle H=(x a+y b)(G+H)$

$r_{1}=\left(\begin{array}{c}x \\ i y\end{array}\right), r_{2}=\left(\begin{array}{l}a \\ b\end{array}\right)$, then $r_{1}\left|r_{2}\right\rangle G+q_{2}\left|r_{1}\right\rangle H=x a(G+H)+i b y(H-G)$

$r_{1}=\left(\begin{array}{c}x \\ i y\end{array}\right), r_{2}=\left(\begin{array}{c}i a \\ b\end{array}\right)$, then $r_{1}\left|r_{2}\right\rangle G+⿶_{2}\left|r_{1}\right\rangle H=i x a(G-H)+i b y(H-G)$

327

$r_{1}=\left(\begin{array}{l}i x \\ i y\end{array}\right), r_{2}=\left(\begin{array}{l}-i a \\ -i b\end{array}\right)$, then $r_{1}\left|r_{2}\right\rangle G+\left[r_{2}\left|r_{1}\right\rangle H=-(x a+y b)(G+H)\right.$

328

329

330

331

332

333

334

335

336

337

338

339

340

341

342

343

344

345

346

347
In the first case, we have a baseline, additive, positive contribution from MOPs to similarity. In cases (5b) and (5c) the contribution from MOPs is lower, relative to that in the baseline case (5a). Finally, in case (5d) the contribution to similarity from MOPs is negative, meaning that the similarity we would expect from MIPs would be reduced, because of MOPs. Note that all the modifications to the role vectors do not alter their norm (i.e., the computations for MIPs are unaffected).

Thus, with complex role vectors, the QP model of basic/analogical similarity predicts a possibility that MOPs will interfere with MIPs, which is a novel prediction from the QP model of basic/analogical similarity. Goldstone's (1994b) SIAM model can also predict such an interference. The QP model and SIAM are not necessarily competing models, as they emphasize different aspects of psychological explanation (the QP model is concerned with the guiding mathematical principles, SIAM is a network model aimed at capturing underlying processes). As noted, Goldstone (1994b) indeed reported a nonsignificant trend where MOPs reduce the overall similarity between two objects. Exploring the reality of such interference effects and identifying the circumstances which make them likely is an exciting (and challenging) direction for further work. For example, perhaps more complex stimuli, for which the different roles have complex relationships with each other (such as those employed in Goldstone, 1994b), make interference effects more likely, as opposed to stimuli with simpler structure and more schematic form (e.g., Larkey \& Markman, 2005). Note, Goldstone's (1994b) own view was that such interference effects arise when the same feature is involved in both a MIP and a MOP.

\section{Further exploring the QP model of basic/analogical similarity}

\subsection{Analytical examination}

In Section 3 we provided an example for how the QP model of basic/analogical similarity can account for MIPs and MOPs (and possible interference effects between the two). Here, first, we consider the formal properties of the model more systematically, with an emphasis on three main findings from Goldstone (1994b): (1) MIPs have more of an impact on similarity than MOPs, (2) MIPS increase similarity, and (3) MOPs increase similarity. Second, having analytically examined the formal sensitivity of the model to these aspects of structural similarity, we provide a computational demonstration of its behavior.

We use the terminology of Smolensky et al. (in press), to make it easier to appreciate the links between our work and theirs. Consider objects composed of $n$ roles (these would be analogous to stimulus components or parts, in the language of basic similarity models) and a set of fillers for each role (a filler is a feature, that is, the way in which a particular stimulus component can appear). Let $\left|r_{1}\right\rangle$, 
$\left.\left|r_{2}>, \ldots,\right| r_{n}\right\rangle$ be $n$-dimensional positive real vectors with unit length representing the roles. Let $\mid f_{i}>$ be a real unit length vector representing the filler associated with role i. A standard simplifying assumption in similarity research is that fillers are orthonormal, so that there is no similarity between the different e.g. features which make up a schematic stimulus. Although we assume filler orthonormality in this section, the QP model does not require it; we discuss the implications of violating this assumption and, also, do not require it in the computational demonstration (note, Goldstone's, 1994b, model incorporates violations of filler orthonormality in terms of a global parameter). Note that, unless stated otherwise, role orthonormality is not assumed (MOPs arise when roles are not orthonormal).

In the quantum similarity model, an object $S$ is represented by the tensor product $r_{1} \otimes f_{1}+r_{2} \otimes f_{2}$ $+\ldots+r_{n} \otimes f_{n}$. The similarity between two objects $S$ and $S^{\prime}$ can be written as the following, up to a normalizing constant:

$$
\begin{aligned}
\operatorname{Sim}\left(S, S^{\prime}\right) & =\left|\left\langle S \mid S^{\prime}\right\rangle\right|^{2} \\
& =\left|\left\langle r_{1} \otimes f_{1}+r_{2} \otimes f_{2}+\ldots+r_{n} \otimes f_{n} \mid r_{1} \otimes f_{1}^{\prime}+r_{2} \otimes f_{2}^{\prime}+\ldots+r_{n} \otimes f_{n}^{3}\right\rangle\right|^{2} \\
& =\left|\sum_{i=1}^{n} \sum_{j=1}^{n}\left\langle r_{i} \mid r_{j}\right\rangle\left\langle f_{i} \mid f_{j}^{\prime}\right\rangle\right|^{2}
\end{aligned}
$$

A MIP between $S$ and $S^{\prime}$ implies there is some role $i$, such that the fillers for the role match, $\left\langle f_{i} \mid f_{i}^{\prime}\right\rangle=1$. A MOP between $S$ and $S^{\prime}$ implies there are roles $i$ and $j$, which are not orthonormal (i.e., $0<\left|<r_{i}\right| r_{j}>\mid \leq 1$; note that $\left\langle r_{i} \mid r_{j}\right\rangle$ could be negative, hence the absolute value is necessary), such that the fillers match, $\left\langle f_{i} \mid f_{j}^{\prime}\right\rangle=1$. In other words, the existence of a MOP implies the conjunction of two things: $\left.\left|\left\langle r_{i} \mid r_{j}\right\rangle\right|\right\rangle 0$ and $\left\langle\mathrm{f}_{\mathrm{i}} \mid \mathrm{f}_{\mathrm{j}}^{\prime}\right\rangle=1$. Also, notice that $\left|\left\langle\mathrm{r}_{\mathrm{i}} \mid \mathrm{r}_{\mathrm{j}}\right\rangle\right| \leq\left|\left\langle\mathrm{r}_{\mathrm{i}} \mid \mathrm{r}_{\mathrm{i}}\right\rangle\right|=1$, because of the assumption that role vectors have unit length. That is, matching roles will have more impact on similarity judgments, than nonmatching roles.

Our first claim is that MIPs have more of an impact on similarity than MOPs. Let $S, S^{\prime}$, and $S^{\prime \prime}$ have $n$ roles. Let $S$ and $S^{\prime}$ have $k \leq n$ MIPs (and no MOPs) and let $S$ and $S^{\prime \prime}$ have $k \leq n$ MOPs (and no MIPs). If different fillers are orthonormal, that is, $\left\langle f_{i} \mid f_{i}\right\rangle=1$ and $\left\langle f_{i} \mid f_{i}^{\prime}\right\rangle=0$ for $f_{i} \neq f_{i}^{\prime}$, then $\operatorname{Sim}\left(S, S^{\prime}\right) \geq$ $\operatorname{Sim}\left(S, S^{\prime \prime}\right)$. The proof is straightforward. Since there are no MOPs in the comparison of $S$ and $S^{\prime}$, all of the fillers for mismatching roles are different. Then, $\left\langle f_{i} \mid f_{j}^{\prime}\right\rangle=0$ for all $i \neq j$ because of filler orthonormality. The presence of MIPs implies there are exactly $k \leq n$ nonzero terms of the form $\left\langle r_{i} \mid r_{i}\right\rangle\left\langle f_{i} \mid f_{i}^{\prime}\right\rangle$ where $\left\langle r_{i} \mid r_{i}\right\rangle=1$ and $\left\langle f_{i} \mid f_{i}^{\prime}\right\rangle=1$. Since there are no MIPs in the comparison of $S$ and $S^{\prime \prime}$, all of the fillers for matching roles are different. That is, $\left\langle f_{i} \mid f_{i}{ }^{\prime \prime}\right\rangle=0$ for all $i$, because of filler orthonormality. The presence of $n$ MOPs implies there are exactly $k \leq n$ nonzero terms of the form $\left\langle r_{i} \mid r_{j}\right\rangle\left\langle f_{i} \mid f_{j}{ }^{\prime \prime}\right\rangle$ where $\left.0<\left|<r_{i}\right| r_{j}\right\rangle \mid \leq 1$ and $\left\langle f_{i} \mid f_{j}^{\prime \prime}\right\rangle=1$. Because $\left.\left|\left\langle r_{i} \mid r_{i}\right\rangle\right| \geq\left|<r_{i}\right| r_{j}\right\rangle \mid$ for all $i$ and $j, \operatorname{Sim}\left(S, S^{\prime}\right) \geq \operatorname{Sim}\left(S, S^{\prime \prime}\right)$.

Without filler orthonormality, we could have situations where two objects have a high similarity without either MIPs or MOPs. Suppose $\left\langle r_{i}\right| r_{j}>\neq 0$ (no MIPs) and we do not have the same feature in the roles $i, j$ (no MOPs). Then, without filler orthonormality, there could be instances when $\left\langle f_{i} \mid f_{j}^{\prime}\right\rangle>0$. Depending on the similarity structure of the features, a large number of (relatively high value) crossterms of this form could exceed the contribution of a small (or zero) number of actual MIPs or MOPs, in a different comparison. This partly illustrates why filler orthonormality is a useful assumption in practical research, since ignoring cross-terms (i.e., ignoring contributions to similarity which are neither MIPs nor MOPs and, instead, arise from similarity between non-identical features) makes similarity computations and modeling more tractable. 
The second claim concerns the impact of MIPs on similarity judgments. We show that increasing the number of MIPs increases similarity. Specifically, suppose $S$ and $S^{\prime}$ have more MIPs than $S$ and $S^{\prime \prime}$ and there are no MOPs. Then, if different fillers are orthonormal, that is, $\left\langle f_{i} \mid f_{i}\right\rangle=1$ and $\left\langle f_{i} \mid f_{i}^{\prime}\right\rangle=0$ for $f_{i} \neq$ $f_{i}^{\prime}$, it will be the case that $\operatorname{Sim}\left(S, S^{\prime}\right)>\operatorname{Sim}\left(S, S^{\prime \prime}\right)$. To prove this, note first that, since there are no MOPs, all of the fillers for mismatching roles are different. That is, $\left\langle f_{i} \mid f_{j}^{\prime}\right\rangle=0$ and $\left\langle f_{i} \mid f_{j}^{\prime \prime}\right\rangle=0$ for all $i \neq j$ because of filler orthonormality. The remaining terms correspond to matching roles and are of the form $\left\langle r_{i}\right| r_{i}>\left\langle f_{i} \mid f_{i}^{\prime}\right\rangle$ for $\operatorname{Sim}\left(S, S^{\prime}\right)$ and $\left\langle r_{i} \mid r_{i}\right\rangle\left\langle f_{i} \mid f_{i}^{\prime \prime}\right\rangle$ for $\operatorname{Sim}\left(S, S^{\prime \prime}\right)$, where $\left\langle r_{i} \mid r_{i}\right\rangle=1$ for all i. A MIP implies the same filler in matching roles. Because we assume the fillers are orthonormal, $\left\langle f_{i} \mid f_{i}^{\prime}\right\rangle=1$ if $f_{i}=f_{i}^{\prime}$ and zero otherwise, likewise for $\left\langle f_{i} \mid f_{i}^{\prime \prime}\right\rangle$. Because $S$ and $S^{\prime}$ have more MIPs than $S$ and $S^{\prime \prime}$, there exist more fillers $f_{i}^{\prime}$ such that $f_{i}=f_{i}^{\prime}$ than fillers $f_{i}^{\prime \prime}$ such that $f_{i}=f_{i}^{\prime \prime}$. Thus, there are more nonzero terms equal to 1 in the calculation of $\operatorname{Sim}\left(S, S^{\prime}\right)$ than $\operatorname{Sim}\left(S, S^{\prime \prime}\right)$.

Eliminating the assumption of filler orthonormality potentially confounds this conclusion. Suppose there are fewer MIPs in comparing $S$ with $S^{\prime \prime}$, than in comparing $S$ with $S^{\prime}$, and no MOPs. In comparing $\mathrm{S}$ with $\mathrm{S}^{\prime \prime}$, it could be the case that there is a large contribution from feature pairs where the roles are identical, but $\left\langle\mathrm{f}_{\mathrm{i}} \mid \mathrm{f}_{\mathrm{i}}^{\prime}\right\rangle \neq 1$ (i.e., no MIP) and $\left\langle\mathrm{f}_{\mathrm{i}} \mid \mathrm{f}_{\mathrm{i}}^{\prime}\right\rangle>0$.

By analogy with the second claim, the third claim is that MOPs increase similarity. That is, suppose $S$ and $S^{\prime \prime}$ have a subset of the MOPs of $S$ and $S^{\prime}$ and there are no MIPs (e.g., ABCD vs. BCXZ compared to $A B C D$ vs. $B C D Z)$. If different fillers are orthonormal, then $\operatorname{Sim}\left(S, S^{\prime}\right)>\operatorname{Sim}\left(S, S^{\prime \prime}\right)$. For the proof, consider that, if there are no MIPs, then all of the fillers for matching roles are different. That is, $\left\langle f_{i} \mid f_{i}^{\prime}\right\rangle=0$ and $\left\langle f_{i} \mid f_{i}{ }^{\prime \prime}\right\rangle=0$ for all $i$ because of filler orthonormality. The remaining terms corresponding to mismatching roles are of the form $\left\langle r_{i} \mid r_{j}\right\rangle\left\langle f_{i} \mid f_{j}^{\prime}\right\rangle$ for $\operatorname{Sim}\left(S, S^{\prime}\right)$ and $\left\langle r_{i} \mid r_{j}\right\rangle\left\langle f_{i} \mid f_{j}{ }^{\prime \prime}\right\rangle$ for $\operatorname{Sim}\left(S, S^{\prime \prime}\right)$ where $<r_{i}\left|r_{j}\right\rangle>0$. A MOP implies the same filler in different roles. Because we assume the fillers are orthonormal, $\left\langle f_{i} \mid f_{j}^{\prime}\right\rangle=1$ if $f_{i}=f_{j}^{\prime}$ and 0 otherwise, likewise for $\left\langle f_{i} \mid f_{j}{ }^{\prime \prime}\right\rangle$. Because $S$ and $S^{\prime \prime}$ have a subset of the MOPs of $S$ and $S^{\prime}$, there exist some $i$ and $j$ such that $f_{i}=f_{j}^{\prime}$ and $f_{i} \neq f_{j}^{\prime \prime}$. (Note that the MOPs of $S$ and $S^{\prime \prime}$ involve the same role vectors as the MOPs of $S$ and $S^{\prime}$, due to the fact the MOPs of $S$ and $S^{\prime \prime}$ are a subset of those of $S$ and $S^{\prime}$.) Therefore, there are more nonzero terms of the form $\left\langle r_{i} \mid r_{j}\right\rangle\left\langle f_{i} \mid f_{j}^{\prime}\right\rangle$ for $\operatorname{Sim}\left(S, S^{\prime}\right)$ than terms of the form $\left\langle r_{i} \mid r_{j}\right\rangle\left\langle f_{i} \mid f_{j}^{\prime \prime}\right\rangle$ for $\operatorname{Sim}\left(S, S^{\prime \prime}\right)$.

The implications for violating filler orthonormality are as above. Here, additionally, we have to take into account the similarity structure between roles, between the compared stimuli. All the MOPs concern terms for which $\left\langle f_{i} \mid f_{j}^{\prime}\right\rangle=1$. In addition, for a MOP, we have $\left\langle r_{i} \mid r_{j}\right\rangle>0$. But the exact magnitude of $\left\langle r_{i} \mid r_{j}\right\rangle$ will depend on role similarity and this may vary from comparison to comparison. It could be the case that a smaller number of MOPs could contribute more to similarity than a greater number of MOPs, if the role similarities for the former are suitably higher. This is why the 'subset' assumption is needed to prove this third claim. For simple, schematic stimuli, it is reasonable to assume that role similarity is flat, that is, all terms of the form $\left\langle r_{i} \mid r_{j}\right\rangle$, which are nonzero, have the same magnitude. In such a case, the 'subset' assumption is not needed and this claim can be proved just on the basis of filler orthonormality.

\subsection{Computational examination}

The analytical demonstration shows that broad level predictions from the QP model are sensible. But how well does the QP model perform, against relevant models? We considered the results from 
Hodgetts, Hahn, and Chater's (2009) Experiment 1. Hodgetts et al. (2009) built on previous empirical studies on analogical similarity (e.g., Larkey \& Markman, 2005) and, moreover, they reported results from a range of relevant models. Their Experiment 1 is the simplest one in their paper and so suitable for this first computational examination of the QP model of basic/analogical similarity.

The results from Hodgetts et al.'s (2009) Experiment 1 consisted of 13 object pairs, with information on the proportion of times each object pair was selected, amongst alternative object pairs, in a forced choice task. Selection was based on the perceived similarity between the objects making up an object pair. Each object was composed of two features (out of three available, denoted $A, B$, and C), each placed in one of two positions (i.e., two roles). Thus, an object pair could be represented as AA/AA, indicating that the same features appeared in both roles, for both objects. Unsurprisingly, this object pair was selected most frequently (91.56\% of all times). By contrast, object pair AB/BB was selected only about half the time. The least selected object pair was AB/CC (23.57\%). Hodgetts et al. (2009) manipulated the physical form of the three features, so that, depending on the condition, $A$ could be any of a circle, a square, or a triangle.

To motivate the parametric choices for the QP model, consider a simple example of computing the similarity between objects, such that Object $_{1}=r_{1} \otimes f_{1}+r_{2} \otimes f_{2}$ and Object $_{2}=r_{1} f_{3}+r_{2} f_{4}$ (for the second object and henceforth we omit the tensor product symbol). Then, Sim(object1, object 2$)=$

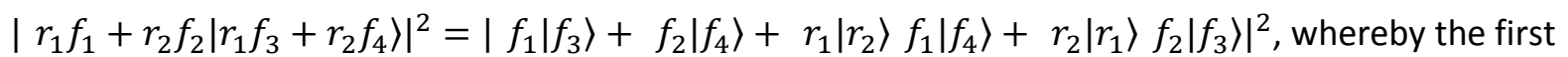
two terms are contributions from MIPs and the latter two from MOPs. Casually inspecting Hodgetts et al.'s (2009) Experiment 1 data, it is clear that there are contributions from MOPs (e.g., the object pair $A B / B A$ was chosen quite frequently; without MOPs, the two objects in the pair would have no similarity). Therefore, we have to allow for non-orthogonal role vectors. Also, the data indicate that the assumption of feature orthonormality is not tenable as well (e.g., $A B / A A$ was not chosen as equally often as AB/BB). Indeed, intuitively, it is unlikely that participants considered a square, circle, and triangle as maximally dissimilar and equally similar to each other.

Based on these observations, we chose to represent roles as non-orthogonal vectors in a two dimensional real space and features as non-orthogonal vectors in a separate two dimensional real space. The restriction to real spaces implies no possibility of interference between the contribution to similarity from MIPs and MOPs, which is reasonable, given our preliminary intuitions for when to expect such effects (Section 3). Note, employing a two dimensional real space for three features implies that it is impossible for the three features to be orthonormal, but limits the overall number of parameters. Overall, the QP model had three parameters, one to characterize feature $A$, one for feature $B$, and one for the first role. Since all vectors are normalized and two-dimensional, a single parameter is needed to characterize any vector. The vector for feature $C$ was fixed to $\frac{1}{\sqrt{2}}\left[\begin{array}{l}1 \\ 1\end{array}\right]$, since what matters is the relative similarity structure of the three feature vectors and we allowed A and B to vary freely; likewise, the vector for the second role was fixed to $\left[\begin{array}{l}0 \\ 1\end{array}\right]$. We allowed the free parameters to vary, so as to optimize the correlation between the similarities between object pairs, computed by the QP model, and the empirically observed choice proportion for each pair.

The best identified correlation was 0.81 and the results are illustrated in Figure 2 (the optimization was carried in Matlab and the code is available from the authors). The 'representational 
distortion' model, favored by Hodgetts et al. (2009), achieved a correlation of -0.95. Hodgetts et al. (2009) also report that the correlation for the Structure Mapping Engine model (SME; Falkenhainer, Forbus, \& Gentner, 1989) was 0.74, the correlation for the SIAM model (Goldstone, 1994b) 0.76, and the correlation which can be achieved by the best linear weighting of MIPs and MOPs 0.77 (this value is inferred from Figure 9 in Hodgetts et al., 2009).

We note the limitations of the QP model. First, achieving the correlation of 0.81 relied on post hoc fitting of three free parameters. By contrast, the representational distortion model had no free parameters (the predictions are based on the specification of a coding scheme), regarding SIAM Hodgetts et al. (2009) employed a recycled set of parameter values from previous work, and SME's application involved no parameters anyway. Thus, the QP model failed to achieve the best fit, even though it was aided by the greatest number of parameters. We can only consider the performance of the QP model as promising, given of course that this is the very first application of the QP model in this domain.

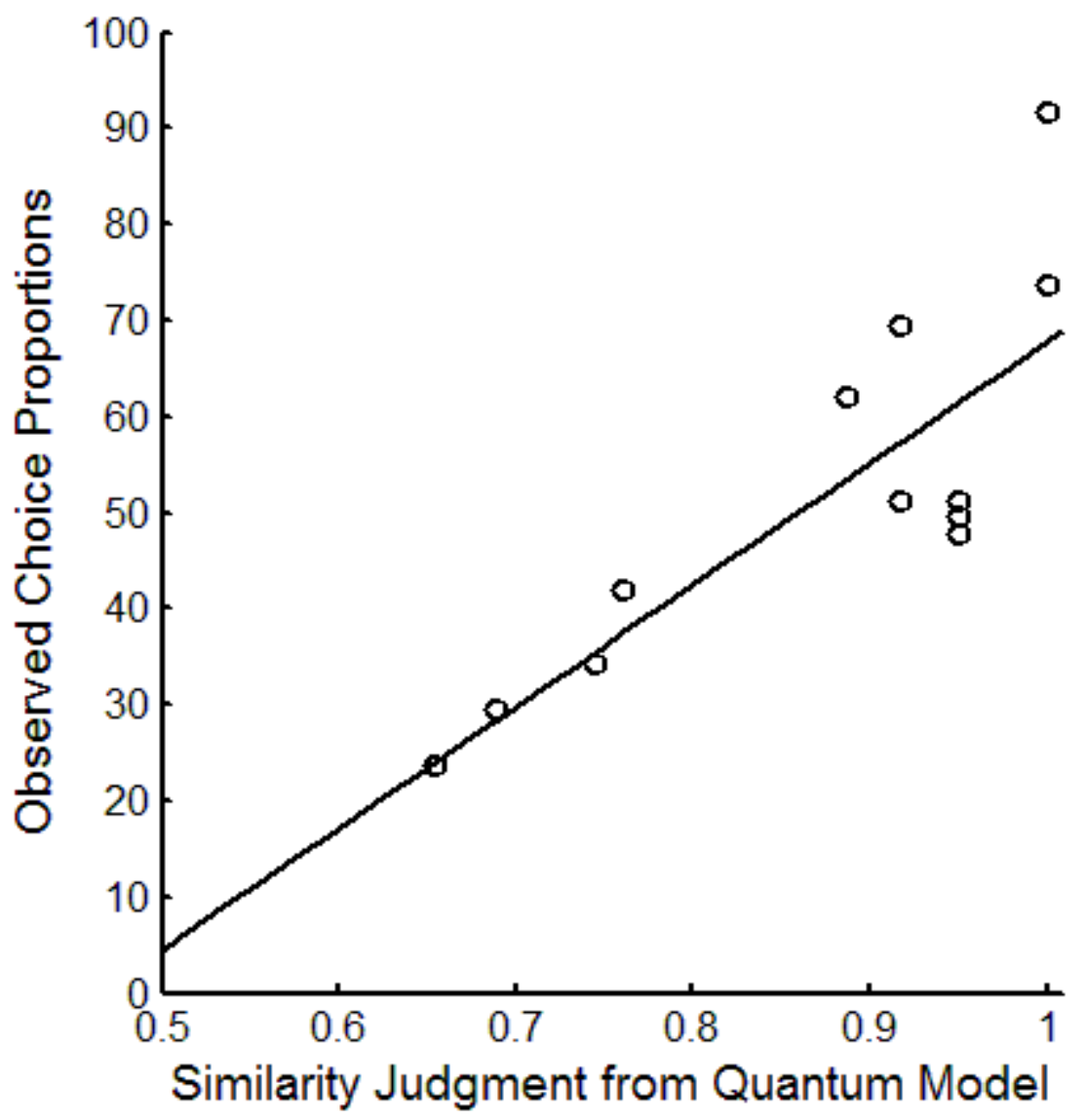

Figure 2. Comparison of the observed choice data from Experiment 1 (Hodgetts et al., 2009) and the best fit of the QP model of basic/analogical similarity, with a regression line.

5. A brief consideration of alternative approaches 
The similarity literature is vast and it is beyond our objective to summarize it in detail (for a review, see Goldstone \& Son, 2005). We consider a limited range of similarity or analogy models, with a view to illustrate some of the points made throughout this paper.

One important motivation for the present paper has been our argument that basic similarity models are ill equipped to handle analogical similarity. This can be illustrated fairly simply. Distancebased models of similarity and feature-based models (such as Tversky's, 1977, model) could, in principle, account for some analogical aspects of similarity (e.g., through the introduction of conjunctive features or dimensions). In simple distance-based models of similarity, the similarity between two entities $A$ and $B$ is given by $\operatorname{Sim}(A, B)=e^{-c \cdot d i s t a n c e}(A, B)$, where $c$ is a constant. In Tversky's (1977) proposal, $\operatorname{Similarity}(A, B)=\theta f(A \cap B)-a f(A-B)-\beta f(B-A)$, where $\theta, a, \beta$ are parameters, $A \cap B$ denotes the common features between $A$ and $B, A-B$ the features of $A$ which $B$ does not have and $B-A$ the features of $B$ which $A$ does not have. In both cases structure could be modeled by conjunctive dimensions or features (e.g., dimension 1 is shape for feature 1 etc.; cf. Goldstone \& Son, 2005), but such a solution is not elegant and appears demanding, in terms of representational resources. Moreover, a distance-based model of similarity could be augmented with a tensor product approach, analogous to Smolensky et al.'s (in press) method. However, without further refinements, such a distance-based model would still have difficulty with Tversky's (1977) empirical findings.

Two important approaches in basic similarity are Krumhansl's $(1978,1988)$ distance-density model and Ashby and Perrin's (1988) general recognition theory. The former model assumes that alternatives lying within dense subregions of psychological space are subject to finer discrimination than alternatives lying in less dense subregions. This implies that the distance (dissimilarity) between two points $A$ and $B$ in psychological space should be affected by the local density around each point, $D(A)$ and $D(B)$. Thus, $d^{\prime}(A, B)=d(A, B)+a D(A)+b D(B)$, where $d(A, B)$ is the standard geometric distance, $a$ and $b$ are parameters, and $d^{\prime}(A, B)$ is the modified distance measure. These ideas are interesting, but it is unclear how to generalize them in the case of structured representations. Ashby and Perrin's (1988) general recognition theory is a probabilistic approach to similarity for perceptual stimuli. Each stimulus (e.g., presented in different trials) can correspond to different points in psychological space, according to a particular probability distribution. Psychological space is divided into response regions, such that within each response region it is optimal to make a particular response. Thus, similarity between two stimuli depends on the overlap between the distribution of perceptual effects for the first stimulus and the optimal response region for the second stimulus. As with Krumhansl's (1978) model, it is unclear how to extend Ashby and Perrin's (1988) approach to take into account the structure of particular stimuli.

We now turn to the converse point: can models of analogical similarity cover Tversky's (1977) key results? Some models of analogical similarity do not consider these results in a detailed way (e.g., Gentner, 1983; Larkey \& Love, 2003; Markman \& Gentner, 1996). An interesting proposal is Taylor and Hummel's (2009) LISA model, which involves a representation scheme analogous to that of Smolensky (1990), but for the crucial difference that roles and fillers are combined additively, rather than multiplicatively. This means that the impact of role similarity is independent of the impact of filler similarity, in establishing an analogy. Regarding computations of similarity, the similarity between two objects $A$ and $B$ reflects a term from MIPs and a term from MOPs. Consider trying to map the structure of a source, $S$, to a target, $T$. Then, the similarity between the two is given by $\sigma_{\text {total }}(T, S)=$ 
$\sigma_{M I P}(T, S)+\sigma_{M O P}(T, S)$, where $\sigma_{M I P}(T, S)$ and $\sigma_{M O P}(T, S)$ is a contribution from MIPs and MOPs, respectively. The first term provides a measure of the extent to which units in $S$ uniquely map onto units in $T$. The second term, $\sigma_{M O P}(T, S)$, is a measure of similarity across all objects and roles in $T$ and $S$, with no regard of whether they map or not. How well does LISA fair with Tversky's (1977) main findings? A key result is that $\operatorname{Sim}($ Korea, China) $>\operatorname{Sim}$ (China, Korea), originally explained as arising, in part, because participants' representation of China would involve more features, than that of Korea. The MIPs term in LISA can be shown to have the form $\frac{\text { features of A uniquely matched to } B}{\text { all features of } A}$ and it can lead to similarity asymmetries, when there are differences in the number of features for $A$ and $B$. Thus, the LISA model can explain the Korea, China asymmetry. Another one of Tversky's (1977) key results, a violation of the triangle inequality, involved similarities amongst three countries, Cuba, Jamaica, Russia. Here, for LISA to accommodate the result in the observed direction, it had to be assumed that Russia was represented in terms of government, Cuba in terms of government and climate, and Jamaica in terms of climate, so introducing a somewhat arbitrary difference in the extent of representations. Notwithstanding the fact that, as far as we can judge, LISA is a very competent model of analogy, we think that its coverage of Tversky's (1977) main results is not fully satisfactory. The specification of LISA involves many architectural choices, which are not always possible to fully constrain. In this way we illustrate what we think is a general feature of models of analogical similarity: the emphasis is on the computational procedures which can lead to the identification of MIPs and MOPs (something currently unspecified in the QP model), not on the algebraic properties of similarity judgments; it is the latter that bears on whether satisfactory coverage with Tversky's (1977) results can be achieved or not.

Finally, another model of analogical similarity, the representational distortion model (Hahn et al., 2003; Hodgetts et al., 2009) could account for violations of symmetry in similarity judgments if, for example, removing elements from a representation can be assumed to be easier (carry a lower information cost) than adding elements (Hahn et al., 2009). Thus, when comparing an informationally simpler object with a more complex one, we should obtain $\operatorname{Sim}($ Complex, Simple) $>$ Sim (Simple, Complex). The model also appears consistent with violations of the triangle inequality, if, for example, one assumes that in e.g. the case of Russia and Cuba the common political system facilitates the 'transformation' of one object to the other. Thus, the representational distortion model potentially encompasses both basic similarity and analogical similarity results.

Where we note shortcomings of the above models, we do not imply criticism, as we examined their application in non-native empirical domains. We simply wish to argue that developing a model encompassing both basic similarity and analogical similarity, as we have attempted to do with the QP model, is a non-trivial task.

\section{Concluding comments}

It is uncontroversial to state that it is desirable to extend specific cognitive models as much as possible. Such extensions inform our understanding of commonalities regarding the underlying cognitive processes, across perhaps seemingly disparate aspects of behavior. They also enable us to understand boundaries in the applicability of particular formalisms and hence the motivation for domain-specific 
mechanisms or principles. It was in this spirit that we set out to explore whether Pothos, Busemeyer, and Trueblood's (2013) QP model of basic similarity could be extended to analogical similarity. We took this to be an interesting challenge, especially because geometric models of basic similarity (such as the QP model) have been thought to be incapable of accommodating structure in similarity judgments (cf. Goldstone \& Son, 2005). As things stand, the QP model of basic/analogical similarity we reported can cover certain aspects of decision making (as in Busemeyer et al., 2011), basic similarity (Pothos et al., 2013), and analogical similarity (the present paper).

We think that the QP model of basic/analogical similarity is, at the very least, a promising candidate for further consideration in the analogical similarity literature. Its formal properties are consistent with expectation, e.g., in relation to the relative impact of MIPs and MOPs. The computational analysis we carried out, based on the results from Experiment 1 of Hodgetts et al. (2009), revealed a decent performance from the QP model, in relation to some standard models of analogical similarity. This was an encouraging preliminary result and recommends further similar computational examinations. e.g., against alternative datasets. Moreover, we identified an interesting novel prediction from the QP model. In standard QP theory, representations are defined over complex, not real, vector spaces. In analogical similarity, employing complex vectors for roles can lead to interference effects between the contributions for MIPs and MOPs. In other words, the QP model predicts (fairly naturally) that there will be some cases when MOPs will conflict with MIPs and so reduce overall similarity (Goldstone, 1994b, states such an implication from his SIAM model too). Developing this prediction in a more specific way is an ambitious objective, though it may be possible to borrow insights from other QP modeling work, for when interference is expected (e.g., Busemeyer \& Bruza, 2012).

In this work we focused on the key challenge for models of analogical similarity of representing structure in a way that it is consistent with MIPs and MOPs, in a certain way. Another key challenge is discovering the appropriate mappings between two objects in the first place (e.g., Goldstone, 1994b; Taylor \& Hummel, 2009). This is an aspect of the QP model of basic/analogical similarity that still needs to be developed. Perhaps some mechanism based on alignment of features (which could be easily implemented in the QP model) may provide a suitable way forward. Somewhat relatedly, we have not considered possible cognitive limitations as the rank of relevant tensors increases (which would happen if the compared objects increase in complexity; cf. Simon, 1955). This has been noted in an alternative approach to analogical similarity (and memory) based on Smolensky et al.'s (in press) ideas (Wiles et al., 1994). An interesting difference between the quantum tensor product approach and traditional ones is that, in the former, the overall required dimensionality can be moderated through the use of incompatible representations. For example, with three binary features, classically we need $2^{3}$ dimensions. In a quantum model, if the features are incompatible, the needed dimensionality could be as low as two (Pothos \& Busemeyer, 2013; Trueblood \& Busemeyer, 2011; cf. Emruli et al., 2013). Exploring more carefully the specific computational resources implied by different modeling approaches is an important priority for further work.

In sum, we think that the QP model of basic/analogical similarity is a promising model of analogical similarity. This encourages us in thinking that the QP principles may provide a firm foundation for understanding aspects of cognition including decision making, basic similarity, and analogical similarity. 


\section{Acknowledgements}

We would like to thank Jerome Busemeyer and James Hampton for their helpful comments. EMP was supported by Leverhulme Trust grant RPG-2013-004 and by Air Force Office of Scientific Research (AFOSR), Air Force Material Command, USAF, grant FA 8655-13-1-3044. JST was supported by NSF grant SES - 1326275. The U.S Government is authorized to reproduce and distribute reprints for Governmental purpose notwithstanding any copyright notation thereon.

\section{$\underline{\text { References }}$}

Aerts, D. (2009). Quantum structure in cognition. Journal of Mathematical Psychology, 53, 314-348.

Aerts, D., \& Gabora, L. (2005). A theory of concepts and their combinations II: A Hilbert space representation. Kybernetes, 34, 192-221.

Ashby, G. F. \& Perrin, N. A. (1988). Towards a Unified Theory of Similarity and Recognition. Psychological Review, 95, 124-150.

Blutner, R., Pothos, E. M., \& Bruza, P. (2013). A quantum probability perspective on borderline vagueness. Topics in Cognitive Science, 5, 1-26.

Busemeyer, J. R. \& Bruza, P. (2012). Quantum models of cognition and decision making. Cambridge University Press: Cambridge, UK.

Casale, M. B., Roeder, J. L., \& Ashby, F. B. (2012). Analogical transfer in perceptual categorization. Memory \& Cognition, 40, 434-449.

Emruli, B., Gayler, R. W., \& Sandin, F. (2013). Analogical mapping and inference with binary spatter codes and sparse distributed memory. In Proceedings of International Joint Conference on Neural Networks (IJCNN), Dallas, Texas, pp 1-88. IEEE Press.

Falkenhainer, B., Forbus, K., \& Gentner, D. (1989). The Structure-mapping engine: Algorithm and examples. Artificial Intelligence, 41, 1-63.

Gentner, D. (1983). Structure-mapping: a theoretical framework for analogy. Cognitive Science, 7, 155170.

Gleason, A. (1957). Measures on the closed subspaces of a Hilbert space. Journal of Mathematics and Mechanics, 6, 885-893.

Goldstone, R. L. (1994a). The role of similarity in categorization: providing a groundwork. Cognition, 52, 125-157. 
670

671

672

673

674

675

676

677

678

679

680

681

682

683

684

685

686

687

688

689

690

691

692

693

694

695

696

697

698

699

700

701

702

703

704

705

706

707

708

709

710
Goldstone, R. L. (1994b). Similarity, interactive activation, and mapping. Journal of Experimental

Psychology: Learning, Memory, and Cognition, 20, 3-28.

Goldstone, R. L, \& Son, J. (2005). Similarity. In K. Holyoak \& R. Morrison (Eds.). Cambridge Handbook of Thinking and Reasoning. Cambridge: Cambridge University Press. (pp. 13-36).

Hahn, U., Chater, N., \& Richardson, L. B. (2003). Similarity as transformation. Cognition, 87, 1-32.

Hahn, U., Close, J., \& Graf, M. (2009). Transformation direction influences shape-similarity judgments. Psychological Science, 20, 447-454.

Haven, E. and Khrennikov, A. (2013). Quantum Social Science. Cambridge University Press: Cambridge, UK.

Hodgetts, C. J., Hahn, U., \& Chater, N. (2009). Transformation and alignment in similarity. Cognition, 113, 62-79.

Hughes, R.I.G. (1989) The Structure and Interpretation of Quantum Mechanics. Cambridge, MA: Harvard University Press.

Hummel, J. E. (2000). Where view-based theories break down: The role of structure in shape perception and object recognition. In E. Dietrich and A. Markman (Eds.). Cognitive Dynamics: Conceptual change in humans and machines. Hillsdale, NJ: Erlbaum.

Humphreys, M. S., Bain, J. D. and Pike, R. (1989). Different ways to cue a coherent memory system: A theory of episodic, semantic and procedural tasks. Psychological Review, 96, 208-233.

Isham, C. J. (1989). Lectures on quantum theory. Singapore: World Scientific.

Khrennikov, A. Y. (2010). Ubiquitous quantum structure: From psychology to finance. Springer.

Knowlton, B. J., Squire, L. R. (1996), Artificial Grammar Learning Depends on Implicit Acquisition of Both Abstract and Exemplar-Specific Information, Journal of Experimental Psychology: Learning, Memory and Cognition, 22, 169-181.

Kolodner, J. L. (1992). An introduction to Case-Based Reasoning. Artificial Intelligence Review, 6, 3-34.

Krumhansl, C. L. (1978). Concerning the applicability of geometric models to similarity data: The interrelationship between similarity and spatial density. Psychological Review, 85, 445-463. 
711 Krumhansl, C. L. (1988). Testing the density hypothesis: comment on Corter. Journal of Experimental

712 Psychology: General, 117, 101-104.

713

714

715

716

717

718

719

720

721

722

723

724

725

726

727

728

729

730

731

732

733

734

735

736

737

738

739

740

741

742

743

744

745

746

747

748

749

750

751

752
Larkey, L. B. \& Love, B. C. (2003). CAB: Connectionist analogy builder. Cognitive Science, 27, 781-794.

Markman, A. B. \& Gentner, D. (1993). Splitting the differences: a structural alignment view of similarity. Journal of Memory and Language, 32, 517-535.

Markman, A. B. \& Gentner, D. (1996). Commonalities and differences in similarity comparisons. Memory \& Cognition, 24, 235-249.

Nelson, D. L., Kitto, K., Galea, D., McEvoy, C. L., \& Bruza, P. D. (in press). How activation, entanglement, and searching a semantic network contribute to event memory. Memory \& Cognition.

Nosofsky, R.M. (1984). Choice, similarity, and the context theory of classification. Journal of Experimental Psychology: Learning, Memory \& Cognition, 10,104-114.

Nosofsky, R. M. (1991). Stimulus bias, asymmetric similarity, and classification. Cognitive Psychology, 23, 94-140.

Pothos, E. M. (2005). The rules versus similarity distinction. Behavioral \& Brain Sciences, 28, 1-49.

Pothos, E. M. \& Busemeyer, J. R. (2013). Can quantum probability provide a new direction for cognitive modeling? Behavioral \& Brain Sciences, 36, 255-327.

Pothos, E. M., Busemeyer, J. R., \& Trueblood, J. S. (2013). A quantum geometric model of similarity.

Psychological Review, 120, 679-696.

Ramscar, M. (2002). The role of meaning in inflection: Why the past tense does not require a rule. Cognitive Psychology, 45, 45-94.

Shafir, E. B., Smith, E. E., \& Osherson, D. N. (1990). Typicality and reasoning fallacies. Memory \& Cognition, 18, 229-239.

Shepard, R. N. (1987). Toward a Universal Law of Generalization for Psychological Science. Science, 237, 1317-1323.

Simon, H. A. (1955). A behavioral model of rational choice. The Quarterly Journal of Economics, 69, 99118.

Smolensky, P. (1990). Tensor product variable binding and the representation of symbolic structures in connectionist networks. Artificial Intelligence, 46, 159-216. 
754 Smolensky, P., Goldrick, M., \& Mathis, D. (in press). Optimization and quantization in gradient symbol

755 systems: a framework for integrating the continuous and the discrete in cognition. Cognitive Science.

756

757 Taylor, E. G. \& Hummel, J. E. (2009). Finding similarity in a model of relational reasoning. Cognitive

758 Systems Research, 10, 229-239.

759

760

Trueblood, J. S. \& Busemeyer, J. R. (2011). A Quantum Probability Account of Order Effects in Inference.

761 Cognitive Science, 35, 1518-1552.

762

763

Tversky, A. (1977). Features of Similarity. Psychological Review, 84, 327-352.

764

765

Tversky, A., \& Kahneman, D. (1983). Extensional versus intuitive reasoning: The conjuctive fallacy in

766 probability judgment. Psychological Review, 90, 293-315.

767

Vokey, J. R., \& Brooks, L. R. (1992). Salience of Item Knowledge in Learning Artificial Grammar. Journal of Experimental Psychology: Learning, Memory \& Cognition, 20, 328-344.

770

771

Wang, Z., Busemeyer, J. R., Atmanspacher, H., \& Pothos, E. M. (2013). The potential of using quantum

772 theory to build models of cognition. Topics in Cognitive Science, 1-17.

773

774

Wiles, J., Halford, G. S., Stewart, J. E. M., Humphreys, M. S., \& Wilson, W. H. (1994). Tensor models: a creative basis for memory retrieval and analogical mapping. In T. Dartnall (ed.), Artificial Intelligence and Creativity, 145-159, Kluwer Academic Publishers. categorization. Psychological Bulletin, 138, 102-125. 